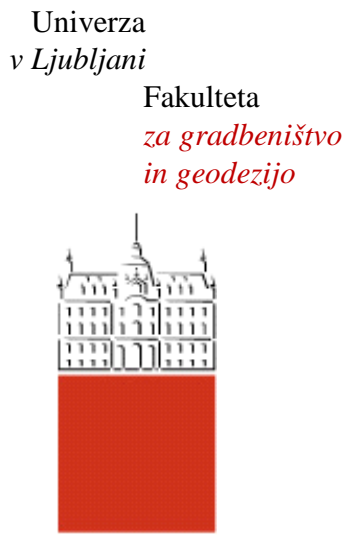

Jamova 2

1000 Ljubljana, Slovenija http://www3.fgg.uni-lj.si/

DRUGG - Digitalni repozitorij UL FGG http://drugg.fgg.uni-lj.si/

Ta članek je avtorjeva zadnja recenzirana različica, kot je bila sprejeta po opravljeni recenziji.

Prosimo, da se pri navajanju sklicujete na bibliografske podatke, kot je navedeno:
University

of Ljubljana

Faculty of

Civil and Geodetic

Engineering

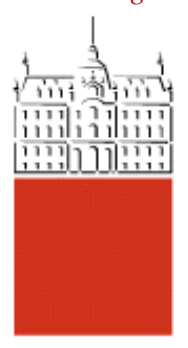

Jamova 2

SI - 1000 Ljubljana, Slovenia

http://www3.fgg.uni-lj.si/en/

DRUGG - The Digital Repository http://drugg.fgg.uni-lj.si/

This version of the article is author's manuscript as accepted for publishing after the review process.

When citing, please refer to the publisher's bibliographic information as follows: 


\title{
Determination of point displacements in the geodetic network
}

\author{
Simona Savšek-Safić ${ }^{1}$, Tomaž Ambrožič ${ }^{2}$, Bojan Stopar ${ }^{3}$, Goran Turk ${ }^{4}$
}

Ms. No. SU/2004/022302

\begin{abstract}
The article describes the procedure for testing the statistical significance of point displacements in the geodetic network as the intermediate stage between the adjustment of respective epochs measurements and an in-depth deformation analysis. The cumulative distribution function of the test statistic, presenting the relation between the displacement and the displacement accuracy, is determined by simulations. On the basis of this cumulative distribution function a critical value of the test statistic for a selected significance level is determined. In the null hypothesis it is assumed that the point is stable. A comparison of the critical value to the test statistic value is made and the actual risk level for rejecting the null hypothesis is estimated. Further on, a practical example of implementing the test in a simulated network is given. The test statistic proved to be simple and applicable: the points with significant displacements were identified successfully.

KEYWORDS: deformation analysis, simulations, hypothesis testing, significant displacements.
\end{abstract}

\footnotetext{
${ }^{1}$ Assistant, University of Ljubljana, Faculty of Civil and Geodetic Engineering, Jamova cesta 2, 1000 Ljubljana, Slovenia, Phone: +386 14768 651, Fax: +3861 4250 704, e-mail: ssavsek@fgg.uni-lj.si

${ }^{2}$ Assistant Professor, University of Ljubljana, Faculty of Civil and Geodetic Engineering, Jamova cesta 2, 1000 Ljubljana, Slovenia, e-mail: tambrozi@fgg.uni-lj.si

${ }^{3}$ Associate Professor, University of Ljubljana, Faculty of Civil and Geodetic Engineering, Jamova cesta 2, 1000 Ljubljana, Slovenia, e-mail: bstopar@fgg.uni-lj.si

${ }^{4}$ Associate Professor, University of Ljubljana, Faculty of Civil and Geodetic Engineering, Jamova cesta 2, 1000 Ljubljana, Slovenia, e-mail: gturk@fgg.uni-lj.si
} 


\section{Introduction}

Basically, deformation analysis is the procedure for determining displacements of assumed stable points and determining significant displacements in geodetic networks. Inaccurate presumptions about assumed stable points in a geodetic network can bring about grave consequences in interpretation of established displacements or when predicting the downfall of buildings. In the process of identification of displacements, the test statistic is very important. A detailed knowledge of deformation analysis methods as well as practical experience are essential for an appropriate interpretation of the estimated point displacements.

In everyday use, the test for determining the statistical significance of a displacement is a function of the point displacement and the respective accuracy. The calculated value is then usually increased by a factor of safety of 3 or 5 or more, which makes the estimation of significant displacements too gross. For the proposed method, simulations of an actual probability distribution function are determined, providing the basis for calculating the right critical value at a chosen significance level. In this way, statistically significant point displacements may be determined far more accurately.

When assessing point displacements, the information on the actual risk of making the error when rejecting the true null hypothesis is very useful and a calculation of this value is advisable. Based on the assumption that the distribution function is established in detail, the suggested test statistic is simple and fit for day-to-day use and refers to the first estimation of the geodetic network. Therefore, it can be carried 
out right after a two-epoch adjustment and accordingly, the need for carrying out the deformation analysis is identified.

\section{Single epoch analysis}

For identification of point displacement by way of geodetic observations, the reference points need to be chosen. Characteristic points on the object are tested for displacements. According to the required accuracy of point displacement determination, the execution of observations must be carried out carefully with proper tools while following standard observational approaches. The observations in the geodetic network are adjusted and the network quality estimated.

Importantly, in networks for displacement identification a network quality estimation is carried out prior to the measurements examining the accuracy, reliability, sensitivity and the cost effectiveness of setting up a network (Caspary 2000). In identification of displacements, network reliability and sensitivity are of primary importance, thus great effort must be made in detecting the presence of undisclosed gross errors. In the planning and optimization phase, the sensitivity of observations needs to be enabled, thereby increasing the probability of detecting outliers.

A well projected network for displacement detection should enable a high degree of detection and elimination of gross errors in observations as well as minimize the effect of potentially undetected outliers influencing the unknowns. Testing the relation between the a posteriori variance $\hat{\sigma}_{0}^{2}$ and the a priori reference variance $\sigma_{0}^{2}$ is 
called the global model hypothesis testing. At the same time, the presence of gross error observations in the network is tested, which is in turn possible only by having a reliable knowledge of the a priori reference variance. In case of incongruence between the observations and the model in the course of the global testing, the Baarda's Data Snooping method for examination, detection and elimination of outliers in observations is introduced. The Pope's Data Screening approach or the Danish approach is used when the a priori reference variance is not reliably known.

After a careful analysis and quality estimation of single epochs, the displacements are estimated and the accuracy of estimating the two-epoch displacements is calculated. In everyday engineering work the difference estimation of point positions between two epochs provides a sufficient amount of information on displacements. This is applicable with a sufficient number of stable points and with displacement that are several times the size of the displacements standard deviations. However, in specific and precise geodynamic research the implementation of a detailed deformation analysis according to one of the several known approaches is essential (i. e. the Delft, Fredericton, Hannover, Karlsruhe, München method etc.).

\section{Testing the significance of displacements}

The basis for displacement determination of a man-made object or any given object on the surface of the earth is to identify the displacements of characteristic points of an object. The points comprise networks, which are monitored in time intervals called epochs that are set out in advance. The point displacements between two epochs can 
be inferred only from identical points, measured in two epochs. However the points are often damaged or they have to be included into the network due to changes of circumstances. Non-identical points are eliminated in the adjustment procedure or with S-transformation, respectively (Mierlo 1978). After the two-epoch adjustment the point displacements and its standard deviations are estimated.

\subsection{Displacement estimation and displacement accuracy estimation}

In geodetic networks set up for determining displacements, the requirement that standard deviations for displacements of geodetic points be provided is very essential. If the estimated displacements are several times the size of the displacement standard deviations, the most probable displacements can be inferred from the differences in point positions. In addition to determining the magnitude and direction of the displacements, the hypothesis testing for the displacemrnt is also necessary. Consequently, these corresponding calculations must be performed.

Point displacements are determined on the basis of comparing point coordinates in two epochs. Let us assume the point coordinates $T(y, x)$ in a plane and time $t$ and $t+\Delta t$. In order to calculate the estimation accuracy of point displacements the covariance matrix of point coordinates for respective epochs must be known. $T_{t}\left(y_{t}, x_{t}\right)$ represents the position of point $T$ in time $t$, and $\boldsymbol{\Sigma}_{t}$ is the corresponding covariance matrix, and $T_{t+\Delta t}\left(y_{t+\Delta t}, x_{t+\Delta t}\right)$ represents the coordinates of point $T$ in time $t+\Delta t$ with the corresponding covariance matrix $\boldsymbol{\Sigma}_{t+\Delta t}$. This can be expressed as 


$$
\boldsymbol{\Sigma}_{T_{t}}=\left[\begin{array}{cc}
\sigma_{y_{t}}^{2} & \sigma_{y_{t} x_{t}} \\
\sigma_{y_{t} x_{t}} & \sigma_{x_{t}}^{2}
\end{array}\right] \quad \text { and } \quad \boldsymbol{\Sigma}_{T_{t+\Delta t}}=\left[\begin{array}{cc}
\sigma_{y_{t+\Delta t}}^{2} & \sigma_{y_{t+\Delta t} x_{t+\Delta t}} \\
\sigma_{y_{t+\Delta t} x_{t+\Delta t}} & \sigma_{x_{t+\Delta t}}^{2}
\end{array}\right] \text {. }
$$

We assume that the coordinates in time $t$ are not correlated with the coordinates in time $t+\Delta t$. Thus, the covariance matrix of coordinates of identical points $y_{t}, x_{t}$, $y_{t+\Delta t}, x_{t+\Delta t}$ can be written as

$$
\boldsymbol{\Sigma}_{T_{t} T_{t+\Delta t}}=\left[\begin{array}{cccc}
\sigma_{y_{t}}^{2} & \sigma_{y_{t} x_{t}} & 0 & 0 \\
\sigma_{y_{t} x_{t}} & \sigma_{x_{t}}^{2} & 0 & 0 \\
0 & 0 & \sigma_{y_{t+\Delta t}}^{2} & \sigma_{y_{t+\Delta x} x_{t+\Delta t}} \\
0 & 0 & \sigma_{y_{t+\Delta t} x_{t+\Delta t}} & \sigma_{x_{t+\Delta t}}^{2}
\end{array}\right] .
$$

The displacement of point $T$ may be evaluated as

$$
d=\sqrt{\Delta y^{2}+\Delta x^{2}}=\sqrt{\left(y_{t+\Delta t}-y_{t}\right)^{2}+\left(x_{t+\Delta t}-x_{t}\right)^{2}} .
$$

Further on the displacement variance is determined by

$$
\sigma_{d}^{2}=\mathbf{J}_{d} \boldsymbol{\Sigma}_{T_{t} T_{t+\Delta t}} \mathbf{J}_{d}^{T},
$$

where the Jacobi matrix $\mathbf{J}_{d}$ equals:

$$
\mathbf{J}_{d}=\left[\begin{array}{llll}
\frac{\partial d}{\partial y_{t}} & \frac{\partial d}{\partial x_{t}} & \frac{\partial d}{\partial y_{t+\Delta t}} & \frac{\partial d}{\partial x_{t+\Delta t}}
\end{array}\right]=\left[\begin{array}{llll}
-\frac{\Delta y}{d} & -\frac{\Delta x}{d} & \frac{\Delta y}{d} & \frac{\Delta x}{d}
\end{array}\right] .
$$


By inserting the equations (1) and (4) into equation (3) we get the representation for displacement variance of point $T$

$$
\sigma_{d}^{2}=\left(\frac{\Delta y}{d}\right)^{2}\left(\sigma_{y_{t}}^{2}+\sigma_{y_{t+\Delta t}}^{2}\right)+2 \frac{\Delta y}{d} \frac{\Delta x}{d}\left(\sigma_{y_{t} x_{t}}+\sigma_{y_{t+\Delta t} x_{t+\Delta t}}\right)+\left(\frac{\Delta x}{d}\right)^{2}\left(\sigma_{x_{t}}^{2}+\sigma_{x_{t+\Delta t}}^{2}\right),
$$

that is used for testing displacements by a test statistic given in equation (6) described in the next section.

\subsection{Determining the distribution function of test statistic with simulations}

In deformation analysis single epochs are usually adjusted as free networks. In this way the best linear unbiased estimation of the unknowns and independence of test statistic regarding the chosen network datum is enabled. After adjusting at least two epochs it is possible to determine the displacement of point $d$ according to equation (2) and standard deviation of displacement $\sigma_{d}$ according to equation (5). Since these two parameters can be calculated prior to a detailed deformation analysis, they are rightly used in the statistical testing.

When estimating displacements the test statistic is often calculated as:

$$
T=\frac{d}{\sigma_{d}}
$$


and compared to the critical value according to the chosen significance level $\alpha$. Point displacements are established with an appropriate probability only when the displacements are significantly larger than the estimation accuracy of displacements.

Assuming that the errors of observations are distributed normally $\varepsilon \sim N\left(0, \sigma^{2}\right)$, then the parameters being the linear functions of the observations $\hat{\mathbf{x}} \sim N\left(\mu_{\hat{\mathbf{x}}}, \sigma_{\hat{\mathbf{x}}}^{2}\right)$ are distributed normally as well. The point displacement is calculated with equation (2). Since $\Delta y$ and $\Delta x$ are calculated as the difference of two normally distributed random unknowns, the $\Delta y$ and $\Delta x$ are distributed normally, too. This, however, is not the case with point displacement $d$, which is a nonlinear function of $\Delta y$ and $\Delta x$. Consequently, it is difficult to analytically determine the form and the type of the distribution of the test statistic (6). The distribution function for the discussed test statistic is therefore determined by simulations (Rubinstein 1981; Savšek-Safić 2002).

The coordinates differences $\Delta y$ and $\Delta x$ are normally distributed random variables with variance-covariance matrix as follows:

$$
\boldsymbol{\Sigma}=\left[\begin{array}{cc}
\sigma_{\Delta y}^{2} & \sigma_{\Delta y \Delta x} \\
\sigma_{\Delta y \Delta x} & \sigma_{\Delta x}^{2}
\end{array}\right]
$$

The standard deviations of coordinates differences in two epochs are calculated as

$$
\begin{aligned}
& \sigma_{\Delta y}=\sqrt{\sigma_{y_{t}}^{2}+\sigma_{y_{t+\Delta t}}^{2}}, \\
& \sigma_{\Delta x}=\sqrt{\sigma_{x_{t}}^{2}+\sigma_{x_{t+\Delta t}}^{2}},
\end{aligned}
$$


where $\sigma_{y_{t}}^{2}, \sigma_{y_{t+\Delta t}}^{2}, \sigma_{x_{t}}^{2}, \sigma_{x_{t+\Delta t}}^{2}$ are coordinate variances of $y_{t}, y_{t+\Delta t}, x_{t}, x_{t+\Delta t}$. The covariance is calculated as:

$$
\sigma_{\Delta y \Delta x}=\sigma_{y_{t} x_{t}}+\sigma_{y_{t+\Delta t} x_{t+\Delta t}}
$$

where $\sigma_{y_{t} x_{t}}$ and $\sigma_{y_{t+\Delta t} x_{t+\Delta t}}$ are covariances of the coordinates in both epochs.

The basic idea for generating a sample of dependent normally distributed random variables is to generate a sample of independent normally distributed random variables and then use a linear transformation to obtain a sample of dependent random variables.

For generating the sample of the normally distributed random variables the Box and Müller approach was applied (Box et al., 1958; Press et al., 1992). Let us assume that $u_{1 i}$ and $u_{2 i}, i=1, \ldots, n$ are samples of two independent and uniformly distributed random variables $U_{1}$ and $U_{2}$, and $n$ is the number of simulations. The sample of two independent normally distributed random variables $Z_{1}$ and $Z_{2}$ is calculated as follows:

$$
\mathbf{z}_{i}=\left[\begin{array}{c}
z_{1 i} \\
z_{2 i}
\end{array}\right]=\left[\begin{array}{l}
\sqrt{-2 \ln u_{1 i}} \sin \left(2 \pi u_{2 i}\right) \\
\sqrt{-2 \ln u_{1 i}} \cos \left(2 \pi u_{2 i}\right)
\end{array}\right], \quad i=1, \ldots, n
$$


For generating a sample of dependent normally distributed random variables a linear transformation is needed. The variance-covariance matrix $\boldsymbol{\Sigma}$ is decomposed by Cholesky decomposition

$$
\boldsymbol{\Sigma}=\mathbf{U}^{T} \mathbf{U}
$$

In our case $\mathbf{U}$ takes the following form

$$
\mathbf{U}=\left[\begin{array}{cc}
\sigma_{\Delta y} & \frac{\sigma_{\Delta y \Delta x}}{\sigma_{\Delta y}} \\
0 & \sigma_{\Delta x} \sqrt{1-\left(\frac{\sigma_{\Delta y \Delta x}}{\sigma_{\Delta y} \sigma_{\Delta x}}\right)^{2}}
\end{array}\right] .
$$

For transformation of a sample of independent normally distributed random variables to a sample of dependent random variables the linear transformation

$$
\mathbf{y}_{i}=\mathbf{U}^{T} \mathbf{z}_{i}, \quad i=1, \ldots, n
$$

is used.

In our case the coordinate differences are generated by the following equations

$$
\begin{aligned}
& \Delta y_{i}=z_{1 i} \sigma_{\Delta y} \\
& \Delta x_{i}=z_{1 i} \frac{\sigma_{\Delta y \Delta x}}{\sigma_{\Delta y}}+z_{2 i} \sigma_{\Delta x} \sqrt{1-\left(\frac{\sigma_{\Delta y \Delta x}}{\sigma_{\Delta y} \sigma_{\Delta x}}\right)^{2}}
\end{aligned}
$$


where it is assumed that the means of $\Delta y$ and $\Delta x$ are zero $\left(\mu_{\Delta y}=\mu_{\Delta x}=0\right)$ and $i=1, \ldots, n$.

The standard deviations of point coordinates in respective epochs vary from point to point. Therefore the distribution function of the test statistic (6) takes on a different form for each point in each two epochs. By using the simulated normally distributed random variables (14), $d$ is calculated using equation (2) and $\sigma_{d}$ using equation (5). Consequently in $n$ simulations, this procedure allows us to determine the empirical cumulative probability distribution function of the test statistic (6) for individual points.

Critical value $T_{c r i t}$ and actual risk $\alpha_{T}$ are determined from obtained empirical cumulative distribution function by the following procedure (see Figure 1):

1. generate coordinate differences $\Delta y_{i}, \Delta x_{i} ; i=1, \ldots, n$ (Equation 14)

2. calculate displacement $d_{i}$ (Equation 2), its standard deviation $\sigma_{d_{i}}$ (Equation 5) and test statistic $T_{i} ; i=1, \ldots, n$ (Equation 6 )

3. form empirical cumulative probability distribution function $F_{T}^{*}$ by sorting $T_{i}$;

$$
F_{T}^{*}\left(T_{i}\right)=\frac{i}{n} ; T_{i} \leq T_{i+1}
$$

4. determinate critical value $T_{c r i t}$ from $F_{T}^{*}: T_{c r i t}=T_{i-\lceil(1-\alpha) n\rceil}$

or

determinate actual risk $\alpha_{T}$ from $F_{T}^{*}: \alpha_{T}=1-\frac{i}{n}$ for such $i$ that $\min T_{i}>T$. 
Figure 1: Empirical cumulative distribution function of the test statistic $T=d / \sigma_{d}$

The test statistic is then tested according to the given null hypothesis and its alternative hypothesis:

$$
\begin{aligned}
& H_{0}: d=0 \text {; the point is stable between two epochs, and } \\
& H_{a}: d \neq 0 \text {; the point has changed its position. }
\end{aligned}
$$

The test statistic (6) is compared to critical value acquired from empirical cumulative distribution function. If the test statistic value is smaller than the critical value at a chosen significance level $\alpha$, then the risk of rejecting the true null hypothesis is too high. Accordingly, it is established that the displacement is not statistically significant. If the test statistic value exceeds the critical value, the risk of rejecting the true null hypothesis is lower than the chosen significance level $\alpha$. Therefore, the null hypothesis is rightly rejected and the statistical significance of the displacement is thereby confirmed.

This decision is supported by calculating the actual risk $\alpha_{T}$ of rejecting the true null hypothesis (the probability of committing Type I Error). Two possibilities are examined:

- $\quad T>T_{\text {crit }}$ i.e. $\alpha_{T}<\alpha$ : the null hypothesis is rejected; the point displacement is statistically significant and

- $\quad T<T_{\text {crit }}$ i.e. $\alpha_{T}>\alpha$ : the null hypothesis is not rejected; the point displacement is statistically non-significant. 
Regarding the actual risk and the consequences of making the wrong decision, it is up to the user to decide upon the risk level of acceptability. As a consequence, a point is thereupon considered as stationary or displaced.

\section{Case example of significant displacement testing in a test network}

In this case a simple test network is established. The displacements of points 1, 2, 3 and 7 are assumed as known. The observations are generated as independent normally distributed with standard deviation of $\sigma_{\alpha}=1^{\prime \prime}$ for angle observations and $\sigma_{s}=5 \mathrm{~mm}$ for distance observations (see Table 1). The geodetic datum of the network is determined as a free network datum. Two epochs are examined with identical types and number of observations (see Figure 2). In the procedure of testing the null hypothesis $H_{0}: d=0$ and significance level $\alpha=5 \%$ are chosen. The empirical cumulative distribution functions are generated by the Premik software (Ambrožič et al 2002) for each point, where the number of simulation is set to 100000 . The simulation is carried out on the basis of 100000 iterations. The existing Premik software was enhanced by adding hypothesis testing which enables the user to determine the statistical significance of the displacement of a particular point. The calculated displacements are compared to the known values. In the following section all the necessary input data for adjustments as well as the adjusted values of point coordinates in single epochs are given.

Table 1: Simulated observations of two epochs 
Table 2: Known displacements of points 1, 2, 3 and 7 between two epochs

Figure 2: Test network and displacements

Table 3: Approximate coordinates equal in both epochs

Table 4: Point coordinates adjustment in a free network adjustment of single epochs

The empirical cumulative distribution function is determined by simulations for the test statistic (6) for each point. Figure 3 shows the cumulative distribution function calculated for Point 2 in a test network. The cumulative distribution function is different for each network point. Regarding Point 2, Figure 3 illustrates the critical value $T_{\text {crit }}=2.384$ at the chosen significance level $\alpha=5 \%$. If the values of $T_{\text {crit }}$ are taken to be 3 or 5 as are generally used as a "rule of thumb", the actual risk is $\alpha_{T(3)}=1.18 \%$ and $\alpha_{T(5)}=0.00 \%$, respectively. Thus the actual risk $\alpha_{T}$ at rejecting the true null hypothesis is set to minimum.

Figure 3: Distribution function of test statistic for Point 2: $T=d / \sigma_{d}=4.724$

Importantly, in the testing procedure one must compare the calculated value of the test statistic to the critical value, $T_{\text {crit }}$ of the test statistic (6) at a chosen significance level. In the test network presented in this paper (Figure 2) the critical values calculated at the significance level of $\alpha=5 \%$ ranged from 2.376 to 2.894 (Table 5). 
Table 5: Significance displacement testing in a test network

As inferred from Table 5, the displacements of statistical significance are undoubtedly present at Points 1 and 7 , since $T>10$. The test statistic value is considerably higher than its critical value, therefore the actual risk $\alpha_{T}$ of rejecting the true null hypothesis is minimal. The suggested test statistic reveals a displacement at Point 2 , since $T>4$ with minimal actual risk of rejecting the true null hypothesis. The actual displacement at Point 3 is not big enough to be statistically significant, since $T<2$. The actual risk of rejecting the true null hypothesis at Point 3 is $\alpha_{T}=24.66 \%$, thus the displacement is not revealed, owing to statistical non-significance. The actual risks for rejecting the true null hypothesis at assumingly stable points 4,5 and 6 exceed $30 \%$ which is substantially more than the chosen significance level $\alpha=5 \%$. Thus, it is not possible to claim that the points had moved.

As illustrated, the critical values for individual points are not equal. Therefore, it is of great importance to determine the distribution function of the test statistic accurately for each network point and to avoid the indiscriminate use of those critical values that are used most frequently.

\section{Conclusion}

A contractor of geodetic works is expected to present not only data on point displacements, but also to provide insurance in terms of the quality of displacement estimation. In addition to the assumed null hypothesis $H_{0}: d=0$ and the chosen significance level $\alpha=5 \%$, the actual risk of rejecting the true null hypothesis is 
crucial. The participation of the commissioning party in the process evaluating the estimated displacements is highly recommended. The decision upon risk acceptability is then in the hands of the commissioner.

As has been shown, test statistic (6) along with the empirical cumulative distribution function are appropriate tools for testing the significance of point displacements in a geodetic network. Since the displacement and its respective accuracy are acquired by a simple method, the suggested procedure is appropriate and provides good results that furnish a good first estimate of the situation in the discussed network. The test example illustrates that the estimation of displacement significance is directly dependent upon the critical value at a chosen significance level $\alpha$. An accurate displacement estimation is achieved only if the critical value is determined according to the actual distribution function of the test statistic. This is a considerable advance with respect to the "rule of thumb" values for $T_{c r i t}=3$ to 5 which were generally used in practical analyses. Having in mind the difficulty level of the assignment and its consequences, the decision must be made whether there is the need for a detailed deformation analysis carried out by one of the known approaches. 


\section{Notation}

The following symbols are used in this paper:

$d \quad=\quad$ displacement of point $T$ between two epochs

$H_{a} \quad=\quad$ alternative hypothesis

$H_{0} \quad=\quad$ null hypothesis

$T \quad=\quad$ actual value of test statistic

$T_{\text {crit }}=\quad$ critical value of test statistic

$F_{T}^{*} \quad=\quad$ empirical cumulative distribution function

$n \quad=\quad$ number of simulation repetitions

$i=$ simulation index

$T_{t}\left(y_{t}, x_{t}\right) \quad=\quad$ coordinates of point $T$ in time $t$

$T_{t+\Delta t}\left(y_{t+\Delta t}, x_{t+\Delta t}\right)=$ coordinates of point $T$ in time $t+\Delta t$

$t \quad=\quad$ time index of 1 st epoch

$t+\Delta t \quad=\quad$ time index of 2 nd epoch

$U_{1}, U_{2} \quad=\quad$ sample of two independent uniformly distributed random variables

$Z_{1}, Z_{2} \quad=\quad$ sample of two normally distributed random variables

$\alpha \quad=\quad$ significance level

$\alpha_{T} \quad=\quad$ actual risk of rejecting the true null hypothesis

$\Delta y, \Delta x=$ coordinates differences between two epochs

$\Delta y_{i}, \Delta x_{i}=$ simulated coordinates differences between two epochs

$\Sigma_{t} \quad=\quad$ variance-covariance matrix of point coordinates in time $t$

$\boldsymbol{\Sigma}_{t+\Delta t}=$ variance-covariance matrix of point coordinates in time $t+\Delta t$ 


$$
\begin{array}{lll}
\boldsymbol{\Sigma}_{T_{t} T_{t+\Delta t}} & =\text { variance-covariance matrix of coordinates of identical points in } \\
& & \text { time } t \text { and } t+\Delta t \\
\boldsymbol{\Sigma} & = & \text { variance-covariance matrix of coordinates differences } \Delta y, \Delta x \\
\sigma_{0}^{2} & =\text { a priori reference variance } \\
\hat{\sigma}_{0}^{2} & =\text { a posteriori reference variance } \\
\sigma_{d}^{2} & =\text { displacement variance of point } T \text { between two epochs }
\end{array}
$$

\section{References}

Ambrožič, T., Turk, G., Stopar, B (2002). "Premik Software.” User manual, ver. 2.0, Internal edition, 2002 (in Slovenian).

Box G.E.P., Müller, M.E (1985). "A note on the generation of random normal deviates.” Annals of Mathematical Statistics, vol. 29, pp. 610-611.

Caspary, W.F (2000). “Concepts of Network and Deformation Analysis.” Kensington, School of Surveying, The University of New South Wales.

Mierlo, J. van (1978). “A testing Procedure for Analysing Geodetic Deformation Measurements.” Bonn, FIG Symposium on Deformation Measurements, 1978.

Rubinstein, R.Y. (1981). "Simulation and the Monte Carlo Method.” New York, John Wiley \& Sons.

Savšek-Safić, S. (2002). "Optimal method for determination of stable points in deformation analysis.” Doctoral thesis. Ljubljana, FGG OGG, 2002 (in Slovenian). 


\section{Figure captions}

Figure 1: Empirical cumulative distribution function of the test statistic $T=d / \sigma_{d}$

Figure 2: Test network and displacements

Figure 3: Distribution function of test statistic for point 2: $T=d / \sigma_{d}=4.724$ 


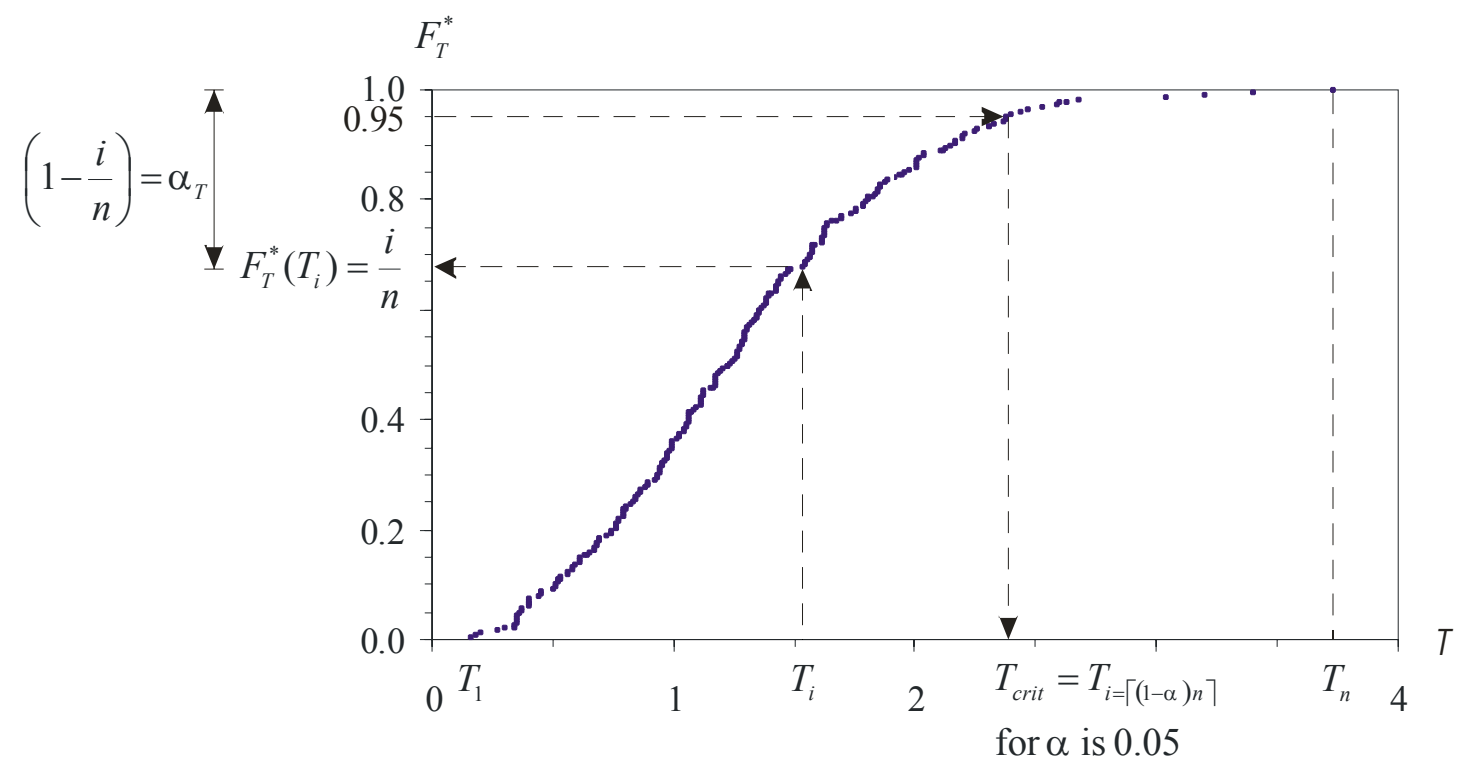

Figure 1: Empirical cumulative distribution function of the test statistic $T=d / \sigma_{d}$ 


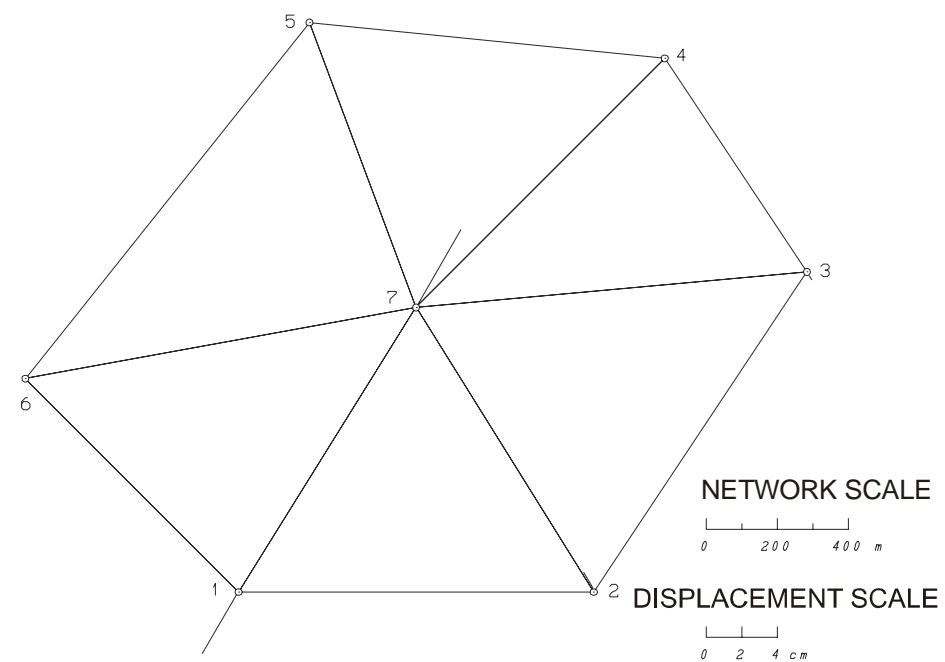

Figure 2: Test network and displacements 


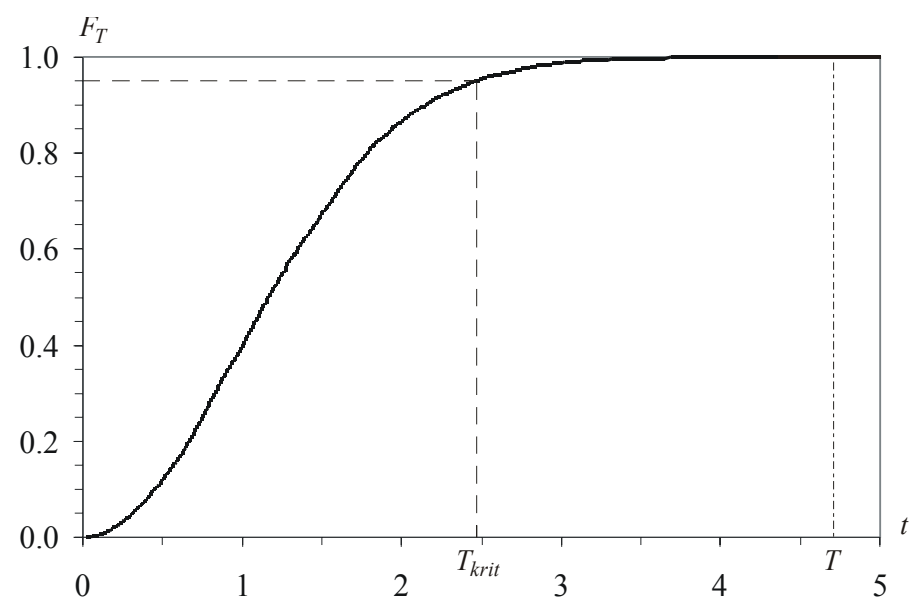

Figure 3: Distribution function of test statistic for Point 2: $T=d / \sigma_{d}=4.724$ 
Table 1: Simulated observations of two epochs

\begin{tabular}{|c|c|c|c|c|c|c|c|c|c|}
\hline \multicolumn{2}{|c|}{ Point } & \multicolumn{4}{|c|}{ Null epoch } & \multicolumn{4}{|c|}{ Epoch 2} \\
\hline \multirow[t]{2}{*}{ From } & \multirow[t]{2}{*}{ To } & \multicolumn{3}{|c|}{ Direction } & \multirow{2}{*}{$\begin{array}{c}\text { Length } \\
{[\mathrm{m}]}\end{array}$} & \multicolumn{3}{|c|}{ Direction } & \multirow{2}{*}{$\begin{array}{c}\text { Length } \\
{[\mathrm{m}]}\end{array}$} \\
\hline & & 0 & , & $"$ & & 0 & ' & $"$ & \\
\hline (1) & (2) & (3) & (4) & (5) & (6) & (7) & (8) & (9) & (10) \\
\hline
\end{tabular}

\begin{tabular}{|c|c|c|c|c|c|c|c|c|c|}
\hline 1 & 6 & 314 & 59 & 58.6 & 848.5203 & 315 & 00 & 08.3 & 848.5437 \\
\hline 1 & 7 & 32 & 00 & 18.4 & 943.4058 & 32 & 00 & 18.0 & 943.4930 \\
\hline 1 & 2 & 90 & 00 & 00.6 & 1000.0017 & 89 & 59 & 48.8 & 1000.010 \\
\hline 2 & 1 & 269 & 59 & 58.1 & 1000.0077 & 269 & 59 & 50.2 & 1000.003 \\
\hline 2 & 7 & 327 & 59 & 41.6 & 943.3963 & 327 & 59 & 50.8 & 943.4170 \\
\hline 2 & 3 & 33 & 41 & 24.9 & 1081.6692 & 33 & 41 & 27.8 & 1081.660 \\
\hline 3 & 2 & 213 & 41 & 23.2 & 1081.6572 & 213 & 41 & 27.7 & 1081.666 \\
\hline 3 & 7 & 264 & 48 & 19.6 & 1104.5400 & 264 & 48 & 28.5 & 1104.507 \\
\hline 3 & 4 & 326 & 18 & 35.0 & 721.1132 & 326 & 18 & 35.0 & 721.1192 \\
\hline 4 & 3 & 146 & 18 & 33.4 & 721.1152 & 146 & 18 & 34.9 & 721.1152 \\
\hline 4 & 7 & 224 & 59 & 59.9 & 989.9525 & 225 & 00 & 00.3 & 989.9073 \\
\hline 4 & 5 & 275 & 42 & 39.1 & 1004.9917 & 275 & 42 & 37.1 & 1004.999 \\
\hline 5 & 4 & 95 & 42 & 37.9 & 1004.9861 & 95 & 42 & 36.1 & 1004.986 \\
\hline 5 & 7 & 159 & 26 & 39.7 & 854.4009 & 159 & 26 & 29.0 & 854.3696 \\
\hline 5 & 6 & 218 & 39 & 36.1 & 1280.6231 & 218 & 39 & 35.9 & 1280.621 \\
\hline 6 & 5 & 38 & 39 & 35.0 & 1280.6242 & 38 & 39 & 34.6 & 1280.626 \\
\hline 6 & 7 & 79 & 41 & 43.7 & 1118.0403 & 79 & 41 & 36.3 & 1118.074 \\
\hline 6 & 1 & 134 & 59 & 59.5 & 848.5338 & 135 & 00 & 10.4 & 848.5325 \\
\hline
\end{tabular}


$\begin{array}{llllllllll}7 & 6 & 259 & 41 & 42.2 & 1118.0366 & 259 & 41 & 36.6 & 1118.068\end{array}$

$\begin{array}{llllllllll}7 & 5 & 339 & 26 & 38.3 & 854.4000 & 339 & 26 & 28.6 & 854.3591\end{array}$

$\begin{array}{llllllllll}7 & 4 & 45 & 00 & 00.9 & 989.9507 & 45 & 00 & 03.6 & 989.8993\end{array}$

$\begin{array}{llllllllll}7 & 3 & 84 & 48 & 21.1 & 1104.5387 & 84 & 48 & 29.6 & 1104.505\end{array}$

$\begin{array}{llllllllll}7 & 2 & 147 & 59 & 40.6 & 943.3984 & 147 & 59 & 50.6 & 943.4008\end{array}$ 
Table 2: Known displacements of points 1, 2, 3 and 7 between two epochs

\begin{tabular}{ccc}
\hline Point & $\begin{array}{c}\text { Displacement }-\mathrm{d} \\
{[\mathrm{mm}]}\end{array}$ & $\begin{array}{c}\text { Azimuth }-v \\
{[0]}\end{array}$ \\
$(1)$ & $(2)$ & $(3)$ \\
\hline 1 & 40 & 210 \\
2 & 12 & 330 \\
3 & 5 & 150 \\
7 & 50 & 30 \\
\hline
\end{tabular}


Table 3: Approximate coordinates equal in both epochs

\begin{tabular}{ccc}
\hline Point & \multicolumn{2}{c}{ Approximate } \\
& \multicolumn{2}{c}{ coordinates } \\
\multicolumn{3}{c}{$y_{0}$} \\
$(1)$ & $(2)$ & $(3)$ \\
\hline 1 & 1000.0000 & 1000.0000 \\
2 & 2000.0000 & 1000.0000 \\
3 & 2600.0000 & 1900.0000 \\
4 & 2200.0000 & 2500.0000 \\
5 & 1200.0000 & 2600.0000 \\
6 & 400.0000 & 1600.0000 \\
7 & 1500.0000 & 1800.0000 \\
\hline
\end{tabular}


Table 4: Point coordinates adjustment in a free network adjustment of single epochs

\begin{tabular}{ccccccc}
\hline \multirow{2}{*}{ Point } & \multicolumn{2}{c}{ Null epoch } & \multicolumn{2}{c}{ Epoch 2 } & \multicolumn{2}{l}{ Coordinate difference } \\
\cline { 2 - 7 } & $\hat{y}_{1}[\mathrm{~m}]$ & $\hat{x}_{1}[\mathrm{~m}]$ & $\hat{y}_{2}[\mathrm{~m}]$ & $\hat{x}_{2}[\mathrm{~m}]$ & $d_{\hat{y}}[\mathrm{~m}]$ & $d_{\hat{x}}[\mathrm{~m}]$ \\
$(1)$ & $(2)$ & $(3)$ & $(4)$ & $(5)$ & $(6)$ & $(7)$ \\
\hline 1 & 999.9988 & 999.9995 & 999.9821 & 999.9599 & -0.0167 & -0.0396 \\
2 & 2000.0013 & 1000.0012 & 1999.9899 & 1000.0085 & -0.0114 & +0.0073 \\
3 & 2600.0037 & 1899.9984 & 2600.0039 & 1899.9942 & +0.0002 & -0.0042 \\
4 & 2200.0004 & 2500.0000 & 2200.0015 & 2500.0007 & +0.0011 & +0.0007 \\
5 & 1199.9988 & 2600.0007 & 1199.9983 & 2599.9966 & -0.0005 & -0.0041 \\
6 & 399.9973 & 1599.9989 & 399.9991 & 1599.9972 & +0.0018 & -0.0017 \\
7 & 1499.9997 & 1800.0013 & 1500.0252 & 1800.0429 & +0.0255 & +0.0416 \\
\hline
\end{tabular}


Table 5: Significance displacement testing in a test network

\begin{tabular}{|c|c|c|c|c|c|c|c|}
\hline \multirow{2}{*}{ Point } & \multicolumn{2}{|c|}{ Simulated displacement } & $\begin{array}{c}\text { Actual } \\
\text { displacement }\end{array}$ & $\sigma_{d}$ & $T$ & $T_{\text {crit }}$ & $\alpha_{T}(\%)$ \\
\hline & $d_{s i m}[\mathrm{~mm}]$ & Displacement & $\begin{array}{c}\text { displacement } \\
\qquad d[\mathrm{~mm}]\end{array}$ & [mm] & & & \\
\hline
\end{tabular}

(1) (2)

(3)

(4)

(5)

(7)

(8)

\begin{tabular}{rrrrrrrr}
\hline $\mathbf{1}$ & 40.0 & yes & $\mathbf{4 3 . 0}$ & 2.7 & 15.931 & 2.382 & $\mathbf{0 . 0 0}$ \\
$\mathbf{2}$ & 12.0 & yes & $\mathbf{1 3 . 5}$ & 2.9 & 4.724 & 2.384 & $\mathbf{0 . 0 0}$ \\
3 & 5.0 & yes & 4.2 & 2.6 & 1.646 & 2.391 & 24.66 \\
4 & 0.0 & no & 1.3 & 2.6 & 0.499 & 2.894 & 88.22 \\
5 & 0.0 & no & 4.1 & 2.8 & 1.466 & 2.376 & 32.66 \\
6 & 0.0 & no & 2.5 & 2.7 & 0.903 & 2.384 & 65.55 \\
7 & 50.0 & yes & $\mathbf{4 8 . 8}$ & 1.9 & 25.838 & 2.387 & $\mathbf{0 . 0 0}$ \\
\hline
\end{tabular}

